\begin{tabular}{llll}
\multicolumn{4}{l}{ Abstract PS-289 Table 1} \\
\hline Outcomes & local-OPC (\%) & national-OPC (\%) & p-value \\
RE-OP & 16 & 14 & 0.76 \\
AC & 4 & 0 & 0.13 \\
WI & 8 & 3 & 0.22 \\
MV & 80 & 88 & 0.34 \\
TPN (<28 days) & 80 & 66 & 0.25 \\
IFALD & 4 & 4 & 1 \\
LOS (<30 days) & 68 & 46 & 0.04 \\
Mortality & 0 & 2 & 1 \\
\hline
\end{tabular}

Background The most commonly performed procedures for SG are operative primary fascial-closure (OPC) and application of a preformed silo with delayed fascial-closure (PS). There is no consensus on optimal management.

Aim To compare our outcomes of SG-newborns after OPC with outcomes from a national cohort (British Association of Paediatric Surgeons-Congenital-Anomalies-Surveillance-System, BAPS-CASS) after OPC or PS.

Methods Retrospective comparison of SG-infants from a tertiary centre (2006-2013) with SG-newborns from BAPS-CASS. We compared baseline characteristics and outcomes: Antenatal diagnosis (AD), in-utero-transfer (IUTR), vaginal delivery (VD), gestational age $\geq 37$ weeks (GA), birthweight $\geq 2500$ g (BW), male gender (MALE), associated anomalies (AA); type/success of surgery, re-operation (RE-OP), abdominal compartment (AC), wound infection (WI), mechanical-ventilation (MV), parenteral nutrition $<28$ days (TPN), intestinal failure associated liver disease (IFALD), length of stay $<30$ days (LOS) and mortality. Datapresentation: Percentage (\%). Data-analysis: Fisher's-Exact-Test $(\mathrm{p}<0.05)$.

Results 25 SG-newborns were compared with 336 newborns from BAPS-CASS. Baseline-characteristics were not significantly different (GA $60 \%$ vs 57\% [p = 0.78], BW 56\% vs 43\% [p = $0.21]$ ), rest not displayed). Significantly more OPC were intended and successfully completed locally than nationally (96\% vs $42 \%$ [p $<0.0001] ; 100 \%$ vs $82 \%$ [p $=0.03])$. Outcomes were:

Compared to national PS TPN and LOS were significantly shorter after OPC locally (80\% vs 48\%[p $=0.004]$; $68 \%$ vs $28 \%[\mathrm{p}<0.0001])$; rest of outcomes not significantly different.

Conclusions Longstanding local experience with OPC positively affects outcomes in SG-infants exceeding some national outcomes irrespective of surgical method.

\section{PS-290 HOW AN INDIVIDUALISED APPROACH COULD CHANGE THE SHORT BOWEL SYNDROME FATE. EXPERIENCE FROM PAEDIATRIC AUTOLOGOUS BOWEL RECONSTRUCTION AND REHABILITATION UNIT}

R Coletta, BA Khalil, A Morabito. Paediatric Autologous Bowel Reconstruction and Rehabilitation Unit (Pabrru), Royal Manchester Children's Hospital, Manchester, Uk

\subsection{6/archdischild-2014-307384.588}

Background and aims Multidisciplinary treatment of short bowel syndrome (SBS) can be considered to be one of the success stories of paediatric surgery. The authors present their experience during the last decade as a specialist unit using improved techniques and individualised approaches in the management of this condition.
Methods Based on data from intestinal rehabilitation program started in 2000, children presenting with SBS were identified. Diagnosis, length of residual bowel, age at definitive surgery, length of bowel pre and post lengthening, and survival were recorded. Data are presented as mean \pm SD.

Results Fifty-three children were identified ( 27 boys, 26 girls). Overall survival was 94\%. Forty-five children required bowel lengthening and 8 children had simple bowel reconstruction while on our protocol. 7 patients was referred after lengthening complication and after our treatment managed postoperatively in other centres outside the UK. Age at definitive surgery was $10.74 \pm 7.9$ months. Residual bowel length in patients' primary treated in our unit was $32.48 \pm 25.89 \mathrm{~cm}$. After bowel lengthening, the bowel length was $69.91 \pm 42.30$ $\mathrm{cm}(\mathrm{p}=0,0001)$. Excluding the 3 patients who died, from 50 patients, $25(50 \%)$ are now off TPN and 25 are weaning HPN (Home Parenteral Nutrition) regimen with a $3.87 \pm 2.10$ nights off.

Conclusions Our series shows improved results not only with survival but also in the number of patients that are off TPN. Individualised approach consisting of both medical and surgical expertise is necessary in the management of these patients. The authors advocate centralisation of short bowel services to experienced centres with multidisciplinary expertise.

\section{PS-291 HETEROTAXIA AND INTESTINAL ROTATIONAL STATUS: IS IT SAFE TO WATCH AND WAIT?}

${ }^{1} \mathrm{P}$ Cullis, ${ }^{1} \mathrm{~S}$ Siminas, ${ }^{2} \mathrm{PD}$ Losty. 'Paediatric Surgery Department, Alder Hey Children's Hospital, Liverpool, UK; ${ }^{2}$ Division of Child Health, University of Liverpool, Liverpool, UK

\subsection{6/archdischild-2014-307384.589}

Background The association of heterotaxia with intestinal rotation anomalies is well documented. Controversy still persists though in regards to the optimal diagnostic strategy, as well as to the benefit of surgical correction of identified rotational abnormalities in patients with no gastrointestinal symptoms, especially given the frequent associated cardiac anomalies. The aim of this study was to test the validity of the watchful wait approach in a series of patients with heterotaxia.

Method A retrospective analysis of the case notes and electronic records of patients diagnosed with heterotaxia syndromes was performed at a single tertiary paediatric referral centre that offers paediatric cardiac surgery services. All patients with situs anomalies, born within the 1st January 1998 to 31st December 2013, were included.

Results 59 patients were identified, of which 13 underwent contrast studies to identify rotational anomalies. Only 2 studies were performed in symptomatic patients (vomiting, failure to thrive). 10 of the studies identified abnormal anatomy, but only 4 patients proceeded to surgical correction. No complications were reported following the Ladd's procedures. There was a single death within 30 days postoperatively, attributed to cardiac failure. Regarding the patients that had no surgery, during the follow-up period (median 9.2 years, range 11- 187 months), no patients presented clinically with malrotation or volvulus.

Conclusions This study supports the 'watch and wait' approach for children with heterotaxia, provided that close surveillance can be achieved. Diagnosis and treatment should be individualised and tailored to each patient's clinical characteristics in order to avoid unnecessary morbidity and mortality. 